\title{
Habilidades sociales y estrategias didácticas para la formación del liderazgo desde la educación básica
}

Tolentino Quiñones, Hermis

Habilidades sociales y estrategias didácticas para la formación del liderazgo desde la educación básica

Revista Educación, vol. 44, núm. 2, 2020

Universidad de Costa Rica, Costa Rica

Disponible en: http://www.redalyc.org/articulo.oa?id=44062184036

DOI: https://doi.org/10.15517/revedu.v44i2.40270

Esta obra está bajo una Licencia Creative Commons Atribución-NoComercial-SinDerivar 3.0 Internacional. 


\title{
Habilidades sociales y estrategias didácticas para la formación del liderazgo desde la educación básica
}

\author{
Social Skills and Educational Strategies for Leadership Training in Primary and Secondary Education
}

Hermis Tolentino Quiñones

Istituto Italiano di Cultura di Lima, Perú

hermis_une@hotmail.com

iD http://orcid.org/0000-0002-9819-1655

\author{
DOI: https://doi.org/10.15517/revedu.v44i2.40270 \\ Redalyc: http://www.redalyc.org/articulo.oa?id=44062184036
}

Recepción: 18 Febrero 2020

Aprobación: 15 Junio 2020

\section{Resumen:}

El presente ensayo tiene como objetivo reflexionar sobre la formación de estudiantes en la educación básica como futuros y futuras líderes. Para tal efecto, en primer lugar, se plantearon las bases teóricas de forma inductiva, es decir, se hizo una descripción general de las cualidades y/o características del liderazgo, y las habilidades que se debe dominar. En segundo lugar, se describieron algunas estrategias y técnicas didácticas para desarrollar, en el futuro líder y la futura lideresa, el aprendizaje complejo, analítico, crítico y reflexivo. Por último, se presentó un modelo de sesión de aprendizaje basado en la piscología de la Gestalt para desarrollar las habilidades sociales y emocionales a través del estudio de caso. Por tanto, se concluyó que la formación del líder y de la lideresa debe iniciarse en la edad escolar empezando por el desarrollo de las habilidades sociales, inteligencia emocional, inteligencia intrapersonal e interpersonal, porque permiten asentar las cualidades y/o características de un buen liderazgo.

Palabras CLAVE: Liderazgo, Formación, Estrategias, Inteligencia, Educación básica.

\begin{abstract}
:
This study reflects on developing skills and abilities in primary and secondary education students with the goal of teaching them to become future leaders. An inductive theoretical framework was first elaborated that involved a general description of qualities or characteristics associated and required skills associated with leadership. Didactic strategies and teaching techniques are described to develop complex, analytical, critical thinking and reflective learning capabilities in future leaders. Lastly, a case study was presented based on a sample class that used Gestalt psychology fundamentals to help develop social and emotional skills. It was concluded that leadership development must begin in primary school, initiating with the development of social skills, emotional intelligence as well intrapersonal and interpersonal intelligence, considered to be qualities that a good leader must be harness and master.
\end{abstract}

KEYWORDS: Leadership, Training, Social Skills, Strategies, Intelligence, Primary and Secondary Education.

\section{INTRODUCCIÓN}

En el mundo, las empresas que tienen visión de futuro contratan y promueven al personal no solamente por las habilidades técnicas que poseen, sino también, por demostrar un domino de las habilidades blandas y sociales, es decir, personas que sean capaces de relacionarse, que sean empáticas, comunicativas y creativas para trabajar en equipo; en pocas palabras, se requiere que sean líderes (Romo, Palacios, Rodríguez y López, 2018), pero la educación de hoy se preocupa poco o nada en la formación de los y las líderes en las escuelas.

Es decir, se ha olvidado que la escuela debe preparar al ser humano para toda la vida, dándole instrumentos que le puedan ayudar a enfrentar las dificultades que se puedan presentar; educándole en la educación emocional: en la inteligencia intra e interpersonal, y romper las barreras tradicionales de lo que es educación/ instrucción. La escuela debería formar al ser humano en todas sus dimensiones: cognitivas, psicológicas, emotivas, creativas, y físicas (Pérez y Filella, 2019).

Por eso, el Perú que no es ajeno a los acontecimientos mundiales, a través del Ministerio de Educación ha elaborado el perfil de egreso de la educación básica regular denominado Plan Nacional hacia el 2020, en el 
cual se detalla, entre otras cosas, la importancia de que el estudiantado sepa tomar conciencia de su persona y de su identidad cultural, incentivando su participación de forma democrática en los acontecimientos de su comunidad y del mundo en general, teniendo siempre presente sus derechos y deberes (Ministerio de Educación del Perú, 2016).

Sin embargo, los avances del gobierno peruano son lentos, por tal motivo, el Plan Nacional hacia el 2020 ha sido ampliado hasta el 2036, porque los objetivos y las metas propuestas no se cumplen (Consejo Nacional de Educación, 2019). Un factor negativo en cuanto a la educación es que no se brinda una formación para la vida de acuerdo con la demanda del mercado laboral; no hay una formación en el sentido empresarial y menos aún en la formación de líderes desde la formación básica (Romo et al., 2018) figura que urge vista las circunstancias políticas y sociales del mundo actual.

Por todo lo expuesto anteriormente, en este ensayo se ha tratado de buscar experiencias documentadas en la formación del estudiantado de la educación básica como líder, sin embargo, estas son, sino nulas, escasas; casi toda la literatura se concentra en la formación del liderazgo pedagógico por su influencia directa en los resultados del proceso de enseñanza-aprendizaje. Por el contrario, existen experiencias de la formación y la importancia de liderazgo en otros campos profesionales, por ello, en el presente trabajo se hará referencia a ellas para sustentar la importancia de la formación de líderes desde los primeros años en la escuela y no esperar hasta la edad adulta.

Así, se tiene el estudio desarrollado por López y Gallegos (2017) donde se demuestra que la participación colaborativa del liderazgo permite obtener resultados positivos en el ámbito académico, influenciando además en la participación del profesorado y los padres y madres de familia. En otra investigación, elaborada en México, se concluye que la participación del consejo técnico escolar permite la inclusión, evitando así la vulnerabilidad social e institucional (Carro, Lima y Carrasco, 2018).

Estas investigaciones sugieren que el liderazgo participativo, donde se requiere la participación de padres, madres, docentes, directores y directoras podría también extenderse al alumnado para así empezar con su propia formación como líderes del futuro y no esperar hasta la edad adulta donde el carácter ya está desarrollado.

Por ello, otra investigación más específica concluye que, en el plan de estudios de algunas escuelas no son consideradas las habilidades blandas o no se les da la debida importancia a pesar de que el mercado internacional y el ambiente competitivo exigen el reforzamiento de estas competencias (Romo et al., 2018).

En la misma línea de estudio, Pérez y Fililella (2019) concluyen que la inteligencia interpersonal e intrapersonal son claves para educar para la vida, permitiendo al estudiantado compenetrarse con sus semejantes; capacidad que debe tener quien es líder o lideresa.

Así también, otros estudios efectuados en Perú dan cuenta de la importancia de la adquisición de las habilidades blandas y comunicativas desde los primeros años en la escuela, demostrando así la relación entre las habilidades comunicativas y el desarrollo de la empatía, la asertividad, la autonomía y el lenguaje social (Cóndor, 2018). La metacognición también tiene relación con las habilidades comunicativas porque permite el conocimiento de lo que uno puede o no puede hacer para saber autorregular el proprio accionar (Nizama, 2018).

Por consiguiente, es necesario que la escuela trabaje en función de las necesidades de la sociedad, organizándose con el personal directivo, docente, los padres de familia, el estudiantado y con la comunidad en general, teniendo prácticas inclusivas y democráticas que puedan romper con la desigualdad social (Valdés y Gómez, 2019).

De lo contrario, se podría llegar a consecuencias negativas como es el caso de una investigación en el sector militar, donde se entrevistó alféreces para obtener reflexiones sobre las oportunidades y el papel que tienen las lideresas, y el dato más resaltante es que aún en un mundo globalizado existen barreras sociales y discriminatorias, incluso por parte de las mismas mujeres que consideraban menos líderesas a las alféreces en contra parte al género masculino (Husain y Muñoz, 2019). 
En consecuencia, para evitar discriminación y prejuicios en cuanto al liderazgo, urge que la escuela esté en armonía y sintonía con la formación cognitiva, artística, deportiva y emocional de estudiantes; articulando el currículo con lo que sucede en el entorno social (Sales, Moliner, Amiama y Lozano, 2018). Esta articulación de la formación del ser humano en todos los aspectos podría ayudar a cimentar las bases de la formación de una persona líder. Porque, en la actualidad, se habla poco o nada sobre la formación de los futuros y las futuras líderes (Burga, 2019; Valdés y Gómez, 2019).

En suma, de la literatura científica presentada se deduce que la preparación del líder y de la lideresa se da en la edad adulta cuando esa debería ser desde los primeros años de la formación académica, en las escuelas. Por ello, nacen dos interrogantes: ¿Cuáles son las características de un buen líder y de una buena lideresa?, ¿Qué estrategias se pueden utilizar para formar a los futuros líderes y lideresas en las aulas de la educación básica? En tal sentido, el presente ensayo tiene como objetivo reflexionar sobre las dos preguntas elaboradas líneas arriba, y para ello se analizarán dos argumentos teóricos y uno práctico: el primer argumento trata sobre el concepto de líder y las cualidades y/ o características que este debe tener; el segundo argumento que se considera es la formación del líder y de la lideresa en las escuelas de educación básica y las estrategias que se podrían utilizar para tal fin. El tercer punto, de corte práctico, consiste en proponer, a modo de ejemplo, un tipo de actividad pedagógica que bien podría dar luces a otras propuestas innovadoras.

\section{Argumentación}

\section{Concepto de líder}

La formación del líder y de la lideresa desde los primeros años en la escuela debe ser uno de los objetivos de la educación, por ello, es imperativo definir qué se entiende por líder y cuáles son las cualidades que debe encerrar esta figura. Chico (1980) explica que el vocablo líder proviene del inglés leader que a su vez deriva del verbo to leard que tiene por significado: acto de guiar y de conducir.

Es así como, en la historia de la humanidad, los y las líderes siempre han jugado un rol emotivo muy importante. En cada época y cultura, el líder y la lideresa ha sido quien ha sabido transmitir tranquilidad y ha sabido guiar un grupo en condiciones de incerteza y peligro (Goleman, Boyatzis y McKee, 2002).

Sin embargo, también existen líderes tóxicos. Para Cubeiro y Gallardo (2008 citados por Reyes, 2014), estos son una gran fuente de desmotivación y pensamientos negativos que afecta a la producción y al clima organizacional. Pero en el presente ensayo se defiende la idea de que solo existe un tipo de líder y es aquel que posee las habilidades para poder dirigir un grupo de personas modificando incluso sus actitudes en pro del bienestar común. El conjunto de líderes posee características especiales que les permiten influenciar a las personas, estimulándolas y motivándolas para trabajar de forma cooperativa y colaborativa con objetivos comunes (López, Fuentes y Moreno, 2018).

Cualidades y/o caracteristicas de una persona lider

No hay un concepto único que defina la figura de líder, pero en este ensayo proponen nueve cualidades y/o características encontradas en muchos estudios, sin embargo, es preciso subrayar que la relación que se presenta no es definitiva y el orden en el cual son descritas no tiene carácter jerárquico:

1. Autoconocimiento, el líder o la lideresa debe tener conciencia de sí mismo; debe saber que sufre una influencia por el marco social en el que está inmerso. Esta influencia se da a través de la socialización y la interacción con diversas situaciones y la pluralidad de los entornos sociales al cual es expuesto (Figueroa y Mijangos, 2018). Sin embargo, no siempre es fácil tener conocimiento del propio ser y de los propios actos, la percepción puede ser errada. Además, se debe discernir de las influencias positivas y negativas.

2. Visionario, debe tener en claro los objetivos y las metas que quiere lograr en el ámbito personal, pero sobre todo en el ámbito social-profesional (Sales et al., 2018). Esto no quiere decir que el 
líder o la lideresa debe abandonar sus propios objetivos, por el contrario, debe mantenerlos y sintonizarlos con los de sus semejantes.

3. Comunicativo, debe saber expresar con claridad sus ideas y formas de pensar, debe saber comunicar su visión para cumplir de forma mancomunada los objetivos y las metas trazadas por el grupo (Sales et al., 2018). La comunicación en cualquier organización es primordial, sin embargo, esta no debe limitarse solo al lenguaje verbal, sino que se debe saber comunicar a través de gestos y actitudes.

4. Democrático, un/a buen/a líder/esa ejerce un liderazgo distribuido (López y Gallegos, 2017) incluyendo en general a toda la comunidad para tomar responsabilidades (Sales et al., 2018); además, tiene actos inclusivos y pone al centro de cada decisión el lado humano (Carro et al., 2018; Valdés y Gómez, 2019).

5. Cooperativo; observa, planifica y decide; el o la líder participa de forma directa en el trabajo para poder alcanzar los objetivos propuestos. Ser líder es demostrar con el ejemplo y es por ello por lo que es la primera persona en colaborar en todo tipo de trabajo que se desarrolla en bienestar de la comunidad (López et al., 2018).

6. Creativo, debe saber analizar la situación y buscar respuestas creativas e inmediatas que puedan favorecer al grupo; debe adaptar su comportamiento de acuerdo con la situación y si es necesario cambiar el estilo de liderazgo (Valdés y Gómez, 2019). Esto no quiere decir que la lideresa o el líder debe ser voluble, por el contrario, debe tener una personalidad firme, de lo contrario se podría malinterpretar su accionar como una forma poco seria de liderazgo.

7. Constante y persistente, asume un alto compromiso con el deber y la responsabilidad (Valdés y Gómez, 2019), además, mantiene una disciplina. Esta cualidad no debe llevarse al extremo opuesto, es decir, no debe convertirse en una obsesión por perseguir objetivos inalcanzables.

8. Investigador, esta característica implica que el o la líder debe poseer un tipo de inteligencia que le permita adquirir conocimientos con base en la observación de la realidad para formular hipótesis y cumplir los objetivos propuesto (Husain y Muñoz, 2019). Esta cualidad es muy importante, pero no debe entenderse como el modo para adquirir conocimientos exclusivamente a través de métodos científicos, sino a través de la propia experiencia.

9. Ético, debe tener bases sólidas ética y moralmente hablando, y debe ser consecuente de lo que dice con lo que hace (López et al., 2018). Esta cualidad debería ser el pilar del líder y de la lideresa, sin embargo, el concepto de lo ético y lo moral puede variar de cultura a cultura, incluso, de persona a persona y en una organización no todos los y las integrantes podrían compartir los mismos principios.

Las nueve cualidades y/o características descritas han sido nombradas y explicadas en los estudios que este ensayo ha consultado, estudios relacionados al liderazgo en varios campos profesionales, sin embargo, cuando se habla de líder se denota una figura ejemplar porque a eso se aspira, a un modelo de líder. Desafortunadamente, se sabe que cumplir con tales cualidades es difícil, incluso, en la historia ha habido líderes negativos y negativas que han llevado al desastre de sociedades enteras.

El lider, la lideresa, ¿nace o se hace?

De acuerdo con Cuervo (2012) el líder o la lideresa nace, pero también se hace, y lo explica a través de un ejemplo: hay personas que nacen físicamente propensas para el atletismo; pero para llegar a ser grandes atletas se requiere de entrenamiento constante; de la misma forma, el o la líder puede formarse teniendo como base las cualidades innatas con las cuales nacen todas las personas.

Entonces, de acuerdo con la propuesta de Cuervo se puede deducir que no todos podrán ser líderes y para serlos debe existir una formación desde los primeros años de vida, tal como lo hace un atleta o una atleta. Es por ello, por lo que la escuela regular básica debería tener como uno de sus objetivos primordiales la formación del líder y de la lideresa o al menos poner las bases para su formación.

Desarrollo de las habilidades social, intrapersonales e interpersonales del lider y de la lideresa 
Considerando la descripción de las nueve cualidades y/o características de líder que se ha hecho anteriormente, se podría decir que las bases para su formación se explican, en un primer nivel, en la teoría de las Habilidades sociales de Goldstein y en la teoría sociocultural de Vygotsky. En un segundo nivel se tienen a las inteligencias múltiples: intra e interpersonal de Howard y la inteligencia emocional de Goleman. A continuación, se detallan cada una de estas teorías:

Goldstein (1980) manifiesta que las habilidades sociales son un conjunto de conductas que los seres humanos utilizan en ciertas circunstancias para hacer frente a los estímulos que son expuestos. Las habilidades sociales de Goldstein pueden ser agrupadas en categorías, las cuales presentan características específicas, desde el saber escuchar e interrelacionarse con los demás hasta saber identificar el enojo en otras personas y saber manejar los propios sentimientos y emociones.

Entonces, se podría decir que las habilidades sociales son las conductas nacientes al momento de interactuar con los semejantes, las cuales permiten poder relacionarse de manera asertiva; estas habilidades se desarrollan desde los primeros años de vida y dependerá de la interacción en la sociedad para fortificarlas (Sales et al., 2018).

En consecuencia, se entiende que el desarrollo o no de las habilidades sociales depende en gran parte del estímulo externo. A menor interacción menos se desarrollarán las habilidades, y es por esta razón que la escuela debe ayudar al estudiantado a desarrollar estas habilidades. Vygotsky (1978), con la teoría sociocultural, plantea que el niño y la niña desarrollan el aprendizaje en medio de la interacción con la sociedad; esto permite que desde los primeros años de vida se vaya adquiriendo las habilidades cognitivas para desenvolverse en la vida social. Es decir, se aprende mientras se interactúa, y es con base en los estímulos internos y externos que el ser humano guiará su forma de comportarse en determinadas situaciones: comunicar sus ideas, a respetar las normas, etc.

Por lo tanto, se puede decir, a modo de ejemplo, que un niño en un ambiente hostil desarrollará habilidades negativas, pero en este ensayo se considera que no es una norma universal, porque, en la historia de la humanidad muchos líderes positivos han surgido, incluso, de ambientes adversos. Claro está que para educar al niño y a la niña como futuro líder y como futura lideresa es necesario que dominen las habilidades sociales e interactúen con sus semejantes. Para poder asentar con mayor claridad los constructos teóricos de Goldstein y Vygotsky, se citan los trabajos de Goleman y Gardner:

En primer lugar, el líder y la lideresa son aquellas personas que tienen una inteligencia emocional de alto nivel, y Goleman (1996) subraya una serie de cualidades que implica este tipo de inteligencia: (a) autoconciencia, es decir, conocerse a sí mismo; (b) autogestión, que implica saber manejar los propios sentimientos; (c) empatía, saber ponerse en los zapatos de los demás; (d) social, saber relacionarse y comunicar sentimientos, emociones e ideas; y (e) motivador, que implica tener un alto grado de motivación intrínseca.

En segundo lugar, en cada persona predomina un tipo de inteligencia (lingüística, musical, lógico matemática, etc.) de acuerdo con la teoría de las Inteligencias Múltiples de Gardner (1994), pero en un líder, en una lideresa debe predominar la inteligencia intrapersonal e interpersonal: la primera consiste en tener una conciencia de quien se es en realidad, reconocer las propias habilidades, destrezas, fortalezas y debilidades para ponerlas en práctica en las diversas circunstancias que se presentan en la vida. La segunda, la inteligencia interpersonal, está relacionada con la capacidad de relacionarse y comprender los sentimientos y actitudes de los demás y saber actuar de forma asertiva.

En conclusión, las habilidades sociales, la inteligencia emocional y la inteligencia inter e intrapersonal permiten al ser humano conocerse y saber relacionarse con su entorno social y actuar de forma armónica con sus semejantes; y esto es un requisito indispensable en la formación del líder y de la lideresa; formación que debe darse desde los primeros años de escuela básica hasta la edad adulta.

Estrategias didácticas para la formación del líder y de la lideresa

Las habilidades sociales, la inteligencia emocional las inteligencias múltiples: inter e intrapersonal como base para la formación del líder pueden ser aprendidas y reforzadas en la escuela, pero ¿de qué manera pueden 
ser enseñadas? Para responder a esta interrogante es necesario analizar algunos conceptos relacionados al constructivismo y al aprendizaje significativo.

De acuerdo con la teoría del constructivismo de Piaget (1935), el ser humano es artífice se su propio aprendizaje y lo hace construyendo sus saberes a través de la propia observación y análisis, es decir, hay un mecanismo interno de reflexión y síntesis. El y la aprendiz, quienes son arquitectos de sus aprendizajes, son un ser con una grande capacidad para tomar sus propias decisiones, deciden qué aprender y qué no, son un ser capaz de tener bajo control sus pensamientos y sentimientos, y al mismo tiempo reconocen aquellos de sus semejantes; es decir, todo su ser es el centro de aprendizaje (Rogers, 1951).

Pero, es necesario aclarar que los aprendizajes son impulsados y desarrollados también por el input externo y elementos como la motivación, el medio a través el cual se presentan los nuevos saberes, la relación con los pares, etc. influyen de forma determinante en el proceso de enseñanza-aprendizaje, ya sea de forma positiva o negativa.

De acuerdo con Ausubel (1983), el nuevo aprendizaje se cimienta sobre las bases del conocimiento previo, o sea, que el aprendizaje no parte de la nada, sino que se tiene en consideración las experiencias adquiridas; a mayor experiencia mayor aprendizaje, mayor retención de la información.

En consecuencia, la nueva información debe ser interiorizada para convertirse en nuevo conocimiento. Y en un mundo globalizado tener acceso a la información es mucho más fácil de lo que era años atrás; hoy en día, casi todo se puede encontrar en Internet, por eso, es de suma importancia saber discernir lo verídico de lo falso y formar a los futuros y futuras líderes en el desarrollo del pensamiento complejo. Para Morin (1999), esto implica un análisis profundo y crítico de la realidad para confrontarla con los conocimientos adquiridos y de esta forma llevarlos a una reflexión y acción. Entonces, como se sustenta en el presente ensayo, la formación del líder y de la lideresa en la escuela implica dominar, en primer lugar, las habilidades sociales y emocionales, cuyo aprendizaje y reforzamiento es posible en las aulas y para ello se hace uso de los diferentes métodos y estrategias didácticas y aquí se sugieren dos: resolución de problemas y estudio de casos.

El primero: resolución de problemas. De acuerdo con Morin (1999), es una metodología propuesta por Ausubel, Bruner, Piaget, Rogers, entre otros, pero de una vigencia actual. Esta estrategia consiste en que el o la docente debe plantear un problema específico en un contexto determinado en función de los objetivos propuesto, después es expuesto al estudiantado para su solución. El alumnado puede hacer uso de cualquier tipo de instrumento y material (internet, por ejemplo) para poder recabar información y dar una posible solución, de este modo se incentiva a estudiantes a poner en práctica los conocimientos adquiridos, elaborar hipótesis y ensayar posibles soluciones. Después, se presentan las conclusiones oralmente y/o por escrito, incluso pude hacerse uso de materiales audiovisuales.

El segundo: el estudio de casos Deriva de la primera estrategia, y consiste en presentar algún tipo de historia (Morin, 1999) con mensajes y/u objetivos específicos donde el estudiantado tendrá que dar una solución y profundizar en el mensaje que se quiere transmitir. La ventaja de esta estrategia es que el alumnado se identifica con facilidad con las historias propuestas, elevando así el grado de motivación y por ende la participación.

En estas dos estrategias es importante que la contextualización sea clara al igual que los objetivos para no guiar a estudiantes a conclusiones erradas o, en todo caso, a conclusiones fuera de contexto. Para la aplicación de estas estrategias es necesario hacer uso de algunas técnicas didácticas, entre estas se proponen: dramatización, role talking, role making, role play, monólogos, debates, talk show, juegos, etc.; pero antes de explicarlas, es importante subrayar que estas técnicas son presentadas a modo de ejemplo. Cada docente debe tener en cuenta la realidad educativa donde enseña para elegir y adaptarlas. A continuación, se detallan las técnicas mencionadas:

La dramatización es una técnica que permite interpretar libretos ya establecidos, sin la necesidad de hacer cambios; esta técnica permite expresar el mensaje a través del lenguaje verbal y no verbal: gestos, movimientos, miradas, distancia corporal, etc.; es de gran utilidad para desarrollar las habilidades socioafectivas (Burga, 
2019). Además, permite analizar los aspectos sociolingüísticos con base en los diversos roles que pueda interpretar cada personaje: social, cultural, psicológico, etc.

De acuerdo con el Nozionario gottodidattico de la Universidad Ca' Foscari de Venezia (2019), el role talking es similar a la dramatización, la diferencia es que el estudiantado puede hacer pequeñas modificaciones o adaptar el libreto al contexto que desean. El role making, en cambio, goza de una mayor libertad, el alumnado crea diálogos, pero sobre la base de las instrucciones del profesor o la profesora. Se puede indicar, por ejemplo, la función de cada participante, que rol debe interpretar (un joven, un anciano, una mujer, un panadero, etc.).

El role play consiste en crear diálogos y cada estudiante tiene una libertad casi absoluta para ello. El o la docente se limita a indicar el tema o el contexto. Para ejecutar esta actividad es importante brindar el tiempo necesario para la creación del diálogo y para la puesta en escena de dramatización. El monólogo, por otro lado, es una buena técnica que permite hacer un análisis más profundo e individual para expresar los propios sentimientos en función del input propuesto. (Nozionario gottodidattico, 2019).

Esta dos últimas técnicas no siempre son apreciadas por el estudiantado porque se siente expuesto, observado y evaluado. Por ello, es importante que el profesorado sepa explicar cuáles son los objetivos que se persiguen.

Otras técnicas muy útiles, sobre todo para presentar situaciones poco comunes o para desarrollar discusión, son el debate y el talk show. El primero consiste en organizar una mesa de discusión donde se presenta un argumento (político, científico, literario, etc.) y diversos panelistas, los cuales son expertos del tema. Cada panelista trata de defender su posición y de convencer a los demás de unirse al propio modo de pensar. Eltalk show en cambio, presenta situaciones y problemas de la cotidianidad (problemas entre hermanos, vecinos, etc.) y los y las panelistas son acusadores, defensores o testigos. El objetivo es tratar de resolver el problema que se presenta decidiendo al final por una culpabilidad o inocencia (Nozionario gottodidattico, 2019).

En ambas estrategias, es importante la planificación que debe hacer el alumnado y preparar el ambiente/ escenario. Además, es importante el tiempo que se proporciona antes, durante y después de la puesta en escena.

También, es posible hacer uso de las actividades lúdicas con el objetivo de trabajar las habilidades sociales (Sales et al., 2018) porque estimulan la creatividad, el respeto por los demás, el respeto de las normas y el equilibrio de las emociones al saber ganar o perder en los juegos. Las actividades lúdicas se basan en los enfoques humanístico-afectivo, comunicativo y en el constructivismo socioafectivo (Caon y Rutka, 2004), y permiten poner en juego la parte cognitiva y emotiva de; además, es altamente motivante e integrador.

\section{Modalidad de trabajo}

Es recomendable usar una modalidad de trabajo en grupo, dando una prioridad al aprendizaje cooperativo porque involucra de modo directo y activo a los o las participantes para poder alcanzar objetivos en común. Con esta modalidad de trabajo, el estudiantado se vuelve experto que ayuda a sus compañeros y compañeras, desarrollan la interdependencia positiva, se motivan entre ellos y ellas y se evalúan a si mismo al elaborar un feedback (Johnson y Johnson, 2015).

Pero, si bien es cierto que el trabajo cooperativo tiene enormes ventajas, no hay que olvidar que el trabajo individual también es importante, sobre todo para elaborar la metacognición que, según Flavell (1985), consiste en autoconocimiento del proceso cognitivo, de lo que uno sabe y es capaz de hacer. Además, la metacognición se relaciona de forma directa con las habilidades significativas, comunicativas y la autorregulación (Nizama, 2018).

Para concluir, se debe recordar que la elección de las estrategias y la modalidad de trabajo al momento de planificar las clases, el y la docente tienen que tener en consideración las necesidades y la realidad del alumnado; se debe conocer los estilos de aprendizaje de cada uno de los y las discentes y de las diversas inteligencias que dominan; también se debe tener en cuenta la edad y el desarrollo cognitivo y, por último, es importante considerar los recursos humanos, materiales y tecnológicos con los cuales se cuenta. 


\section{Propuesta didáctica para el desarrollo de una clase en la formación del lider y de la lideresa}

En la formación del líder y la lideresa, principalmente en niños, niñas y adolescente, es importante la figura de un, una mentora como ejemplo de vida. Este modelo puede ser una persona que haya logrado alcanzar sus propias metas, que haya contribuido con el bienestar de la sociedad, una persona que haya sido capaz de disipar prejuicios y haya superado, en su forma de pensar, las barreras de la discriminación (Gaete, Álvarez y Ramírez, 2019; Husain y Muñoz, 2019).

Por eso, este ensayo sugiere como ejemplo aplicar el estudio de casos sobre la vida y obra de algunos personajes importantes que hayan demostrado ser buenos y buenas líderes. Estos personajes deben abarcar todas las disciplinas posibles: héroes nacionales, escritores, deportistas, artistas, médicos, etc. además, se debe considerar en presentar un numero equilibrado entre personajes femeninos y masculinos.

A modo de ejemplo, se presenta algunas indicaciones de cómo podría organizarse una clase de entre 90 y 100 minutos sobre la vida y obra de algún personaje. Para ello, se utilizará una estructura basada en la psicología de la Gestalt, es decir, desde lo general a lo específico. Este modelo presenta cinco partes, a continuación, se detallan brevemente cada una de ellas:

Motivación: se recomienda presentar alguna foto o frase para extraer los saberes previos del estudiantado y guiarlo a través de preguntas, lluvia de ideas, etc. El trabajo puede hacerse con toda la clase y de forma oral.

Presentación: presentar la biografía de algún personaje, ya sea por escrito, audio/video. Hacer una lectura individual y dirigir la comprensión del texto de forma general a través de preguntas verdadero/falso, sí/no, etc.

Análisis: analizar algunos aspectos de índole lexical (si corresponde), para luego pasar a los análisis significativos de la vida del personaje elegido. Se puede hacer un análisis desde el punto de vista de los pensamientos, obras y logros. O también se puede hacer un análisis histórico social, etc.

Reflexión: se puede pedir al alumnado hipotizar sobre las motivaciones que tuvo el personaje que se estudia para llegar a ser considerado líder. Es posible también identificar, o al menos, hacer hipótesis sobre qué habilidades sociales dominaba este personaje.

Sintesis: en esta fase cada estudiante, después de haber analizado y reflexionado sobre la vida y obra de algún personaje, tendrá que hacer uso de lo adquirido, desde el punto de vista de las habilidades sociales, intra e interpersonales a través de actividades como la dramatización, role play, etc.

En fin, se puede hacer un sin número de actividades, todo dependerá de la creatividad del personal docente para programar una clase y hacer partícipes activos al grupo de estudiantes. Por ello, es de suma importancia poder distinguir los objetivos para organizar la sesión y elegir las estrategias y las técnicas didácticas. Se anexa el desarrollo de una sesión de aprendizaje sobre un poeta peruano como ejemplo. (Véase Apéndice donde es posible encontrar, además, un enlace para ver el material didáctico original).

\section{Conclusiones}

La sociedad, frente a los cambios que sufre en el ámbito científico, tecnológico, cultural y sobre todo político, requiere de líderes bien formados y comprometidos con el bienestar de la población. Pero, tal formación no debe empezar en la edad adulta cuando se tiene la intención de (o se va a) ocupar algún cargo, sino que debe ser un aprendizaje y una preparación constante, que debe empezar en la escuela, desde la educación básica.

Y para ello, es necesario que la escuela prepare al estudiantado, como futuros y futuras líderes, en el manejo de las habilidades sociales, emocionales, intrapersonales e interpersonales; porque, son el cimiento para la formación de los y las líderes.

Además, un buen líder y una buena líder deben desarrollar el pensamiento complejo, y esto se puede concretar a través del aprendizaje basado en la resolución de problemas y el estudio de casos; los cuales permiten desarrollar habilidades críticas, reflexivas y propositivas.

Por tal motivo, se cree conveniente, iniciar la formación de los futuros y las futuras líderes analizando biografías de personajes resaltantes porque son puntos de referencia que el alumnado valora. 


\section{Recomendaciones}

1. Ejecutar talleres para la formación de líderes en las escuelas.

2. Programar sesiones de aprendizaje para desarrollar y potenciar en cada estudiante las habilidades sociales, emocionales, interpersonal e intrapersonal, a fin de cimentar las bases de los futuros y futuras líderes.

3. Incentivar el aprendizaje complejo a través de estrategias y técnicas didácticas donde el estudiantado pueda hacer uso de sus conocimientos cognitivos y emocionales.

4. Elaborar reportes sobre la funcionalidad o no de los talleres para luego difundirlos en la comunidad educativa: directivos, docentes, padres, madres de familia y la comunidad en general.

\section{RefEREnCias}

Ausubel, D. (1983). Psicología de la educación, Un punto de vista cognitivo. México: Trillas.

Burga, A. (2019). La dramatización como estrategia para formar líderes en los estudiantes de quinto grado de una institución educativa de nivel primario en Hualgayoc. (Tesis de maestría). Universidad César vallejo, Perú.

Caon, F. y Rutka, S. (2004). Teoria della glottodidattica ludica. Venezia: Ca' Foscari-FILIM. Recuperado de https:// www.itals.it/sites/default/files/Filim_glotto_ludica_teoria.pdf

Carro, A., Lima, J. y Carrasco, M. (2018). Los consejos técnicos escolares para la inclusión y equidad educativa en la educación básica de Tlaxcala, México. Revista Electrónica Educare, 22(1), 1-30. doi: https://doi.org/10.15359 /ree.22-1.8

Chico, P. (1980). Formación de lideres. Madrid: C. S., Burgos.

Cóndor B. (2018). Habilidades sociales y habilidades comunicativas en los niños de 04 y 05 años en la Institución Educativa $N^{\circ} 160$ "Mi pequeño mundo" de la provincia de Junín, 2018 (Tesis de maestría). Universidad César Vallejo, Perú. Recuperado de: http://repositorio.ucv.edu.pe/handle/UCV/37600

Consejo Nacional de Educación. [CNE] (2019). Proyecto Educativo Nacional al 2036. Perú. Recuperado de http://r epositorio.minedu.gob.pe/handle/MINEDU/6563

Cuervo, J. (2012). Mejor liderar que mandar. Barcelona: Libros de cabecera.

Figueroa, D. y Mijangos, J. (2018). Formación pedagógica y construcción de identidad en líderes educativos comunitarios del CONAFE. Revista Electrónica Cientifica De Investigación Educativa, 4(1), 375-392. Recuperado de https://www.rediech.org/ojs/2017/index.php/recie/article/view/303

Flavell, J. (1985). El desarrollo cognitivo. Madrid: machado.

Gaete, R., Álvarez, J. y Ramírez, M. (2019). Reflexione y experiencias de profesoras investigadoras mexicanas sobre el techo de cristal. Revista Calidad Educativa, (50), 457- 491. doi: https://doi.org/10.31619/caledu.n50.494

Gardner, H. (1994). Inteligencias múltiples. Barcelona: Paidós.

Goldstein, A. (1980). Habilidades sociales y autocontrol en la adolescencia. Barcelona: Martines Roca.

Goleman, D. (1996). Inteligencia emocional. Barcelona. Editorial Kairos.

Goleman, D., Boyatzis R. y McKee, A. (2002). Essereleader. Milán. Editorial Bur.

Husain, S. y Muñoz, L. (2019). Caracterización del liderazgo femenino en ámbitos militares. Reflexiones a partir de las experiencias de las alféreces en su fase de mando. Cadernos Pagu, (56), 1-29. doi: https://doi.org/10.1590/ 18094449201900560009

Johnson, D. y Johnson, R. (2015). El aprendizaje cooperativo en el aula. Buenos Aires: Editorial Paidós.

López, P. y Gallegos V. (2017). Liderazgo distribuido y aprendizaje de la matemática en escuelas primarias: el caso de Chile. Perfiles educativos, 39(158), 112-129. Recuperado de https://doi.org/10.22201/iisue.24486167e.2017.1 58.58786 
Hermis Tolentino Quĩ̃ones. Habilidades Sociales y estrategias didácticas Para la formación Del LiD...

López, J., Fuentes, A. y Moreno, A. (2018). El liderazgo efectivo en los centros concertados de naturaleza cooperativa: Percepciones de sus docentes. Actualidades Investigativas en Educación, 18(3), 318-339. doi: https://revistas.uc r.ac.cr/index.php/aie/article/view/34195

Ministerio de Educación [MINEDU]. (2016). Curriculo Nacional de la Educación Básica. Perú: Recuperado de http s://bit.ly/3dMRz5A

Morin, E. (1999). Los siete saberes necesarios para la educación del futuro. Francia: UNESCO. Recuperado de https:/ /bit.ly/2SB7pr 1

Nizama, P. (2018). Metacognición y habilidades comunicativas en los estudiantes de avanzado del Centro de Educación Básica Alternativa Alfonzo Ugarte, Callao, 2018 (Tesis de maestría). Universidad César Vallejo, Perú. Recuperado de http://repositorio.ucv.edu.pe/handle/UCV/27352

Nozionario gottodidattico (2019). Definiciones de terminaologia didáctica. Venezia: Università Ca’ Foscari di Venezia. Recuperado de https://itals.it/nozion/nozionf.htm

Pérez, N. y Filella, G. (2019). Educación emocional para el desarrollo de competencias emocionales en niños y adolescentes. Praxis y Saber, 10(24), 23-44. doi: https://doi.org/10.19053/22160159.v10.n25.2019.8941

Piaget, J. (1935). El lenguaje y el pensamiento en el niño. Madrid: Morata.

Reyes, R. (2014). Liderazgo tóxico. Boletín de investigación y postgrado. Colombia: Centro de investigación y postgrado de la Universidad Privada Rafael Belloso Chacín. Recuperado de: http://www11.urbe.edu/boletines/postgrad $\mathrm{o} / \mathrm{p}=1185$

Rogers, C. (1951). Client centered-therapy: its current practice, implications and theory. Londres: Constable.

Romo, J., Palacios, P., Rodríguez, C. y López, C. (2018). Reforzamiento de las competencias blandas en la acreditación ABET para la formación de líderes transformadores. Revista electrónica Anfei Digital, 4(8), 1-11. Recuperado de http://anfei.org.mx/revista/index.php/revista/article/view/430

Sales, A., Moliner, O., Amiama, J. y Lozano, J. (2018). Escuela incluida recursos y estrategias para la participación ciudadana. Revista Mexicana de Investigación Educativa, 23(77), 433-458. Recuperado de https://bit.ly/39H8 YKT

Valdés, R. y Gómez, I. (2019). Competencias y prácticas de liderazgo escolar para la inclusión y la justicia social. Perspectiva Educacional, 58(2), 47-68. Recuperado de https://bit.ly/2vOwF55

Vygotsky, L. S. (1978). El desarrollo de los procesos psicológicos superiores. Barcelona: Editorial Crítica.

\section{Apéndice}

Ejemplo de cómo programar una clase para desarrollar la formación del liderazgo en el aula. Para poder ver el formato libro con gráficos e imágenes use el siguiente enlace: https://www.flipsnack.com/proftolentino/ histortias-que-inspiran.html

\section{1. ¿Quién es?}

Lee el siguiente texto sobre la biografía de César Vallejo y complétalo con las palabras sugeridas. Después, verifica tus respuestas con un compañero. ;Ojo! Una palabra no se usa: hermanos - patria - poeta - hogar viernes

César Abraham Vallejo, el más grande peruano nació en Santiago de Chuco en 1892, pero murió lejos de su , un

Mendoza. Tuvo diez santo de 1938, en la ciudad de Paris. Sus padres fueron Francisco Vallejo y Maria de los Santos

\section{2. ¿Cómo era Vallejo?}

Observa las fotos de Vallejo y junto a un compañero describan lo que observan y traten de hacer una hipótesis sobre el carácter del poeta. 


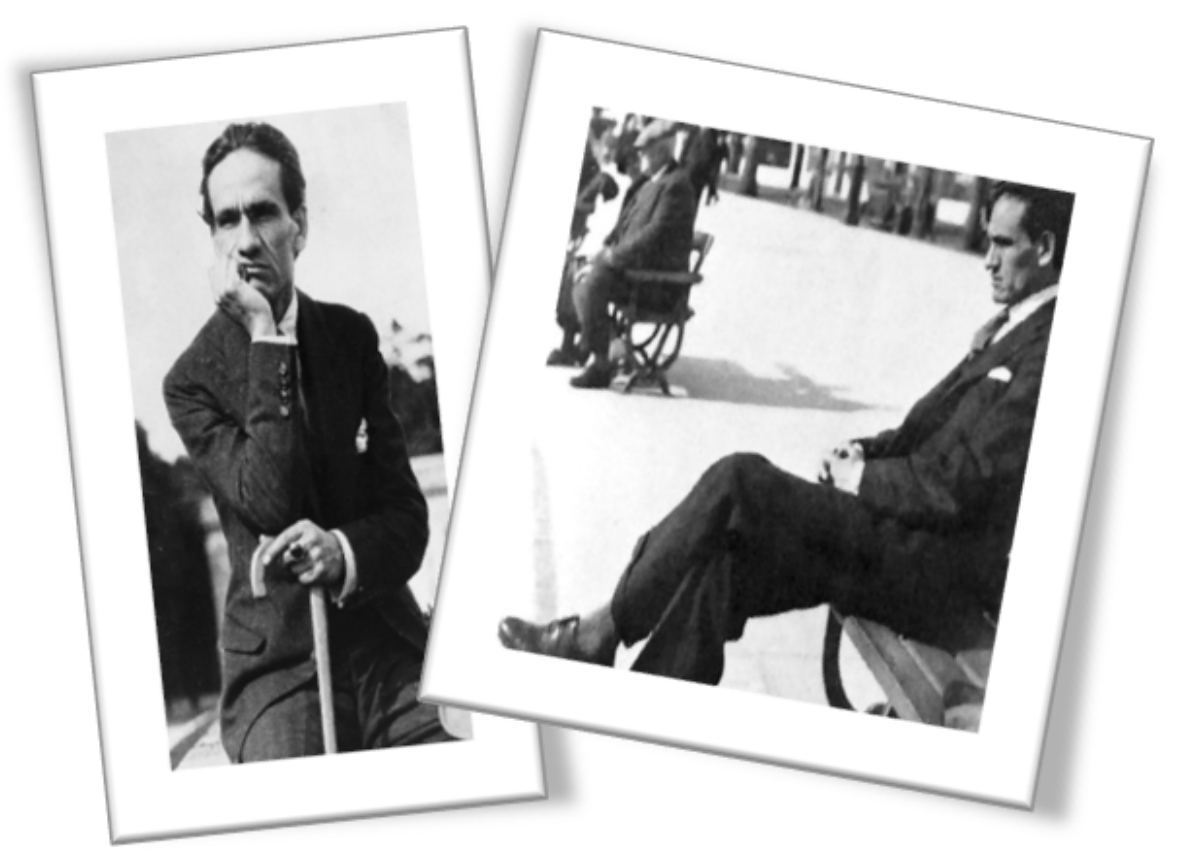

\section{La biografía}

Lee la biografía de César Vallejo y elige entre las dos palabras en negrita. Después, verifica tus respuestas con un compañero.

César Abraham Vallejo, el más grande poeta peruano nació en Santiago de Chuco en 1892, pero murió lejos de su patria, un viernes santo de 1938, en la ciudad de París. Sus padres fueron Francisco Vallejo y María de los Santos Mendoza. Tuvo diez hermanos.

Estudió la primaria en su tierra y la secundaria en Huamachuco, hacia fines/inicios de 1908. Ingresó en 1913 a la Universidad de La Libertad (Trujillo), donde se graduó con una tesis sobre El romanticismo en la poesía castellana.

En 1918 viajó a Lima/Huancayo para estudiar un doctorado en la Facultad de Letras de la Universidad Nacional Mayor de San Marcos. Ese año publicó/escribió su primer libro titulado Los Heraldos Negros.

En 1920, el poeta fue detenido injustamente y llevado a la cárcel de Santiago de Chuco, donde permaneció tres meses/años. Los periódicos de aquella época defendieron su inocencia y gracias al trabajo de su abogado salió libre.

Tras las rejas escribió/leyó Escalas melografiadas. Se dice que este texto sirvió de inspiración para la construcción literaria de Trilce (1922).

César Vallejo, considerado el representante máximo del vanguardismo en el Perú, deseaba viajar a Europa/ Asia y lo hizo en 1923. Estuvo por diversas ciudades europeas como París, Madrid, Moscú, Budapest, Bruselas y Berlín. Permaneció en el Viejo Continente 5/15 años y se casó con la francesa Georgette Philipard.

Lejos de nuestro país escribió Rusia en 1931, Reflexiones al pie de Kremlin, así como una obra de poesía/ teatro llamada Lock-out. También escribió una novela sobre la explotación de una comunidad de indios titulada Tungsteno.

Vallejo careció de recursos económicos/inspiración en Europa. Al principio, vivió de artículos periodísticos y ensayos que enviaba a distintas revistas del país.

Después de su muerte en París/Italia, el 15 de abril de 1938, se publicaron, entre otros, Poemas Humanos y España, aparta de mi este cáliz.

César Vallejo fue un poeta que corregía poco/mucho sus textos. Casi nunca estuvo satisfecho con lo que hacía. 
4. Leamos: verdadero o falso

Lee nuevamente el texto y responde con verdadero $(V)$ o falso $(F)$.

a) Cesar Vallejo fue llevado injustamente a la cárcel. ( )

b) Escribió varios en vario géneros literarios. ( )

c) Murió en Perú. ( )

d) Buscaba la perfección en sus obras. ( )

e) Perteneció a una familia numerosa. ( )

\section{5. ¡Sorprende!}

¿Qué es lo que más te ha impresionado de la vida de César Vallejo? Escribe en el cuaderno tu respuesta (breve). Después, trabaja en pequeños grupos, cada uno da a conocer su punto de vista.

6. Ventajas y desventajas

En la última parte de la biografía de Vallejo se lee lo siguiente:

César Vallejo fue un poeta que corregia mucho sus textos. Casi nunca estuvo satisfecho con lo que hacía.

Cuales crees que puedan ser los aspectos positivos y negativos de la forma de ser del autor.

7. iEntrevista a Vallejo!

Trabaja con un compañero. Uno es César Vallejo y el otro un periodista. Se debe realizar una entrevista sobre la vida del poeta, particularmente sobre el periodo en la cárcel y en Europa. Usen la creatividad para las preguntas y respuestas, después escenifiquen la entrevista.

8. Carta a Vallejo

Escribe una carta a Vallejo; en ella debes: comentarle cual es el aspecto que más te ha impresionado de su vida; cuéntale también, cuáles son tus sueños y ambiciones. Si deseas puedes preguntarle sobre sus obras o decirle que has leido sus poesias.

\section{GUÍA PARA EL DOCENTE}

\section{Datos generales}

Recursos: Fotocopias de la separata, cuadernos o hojas, lapiceros, papelotes, plumones, etc.

Objetivos:

- Formar las bases de los futuros líderes.

- Desarrollar las habilidades sociales, interpersonales e intrapersonales en los estudiantes de la educación básica.

- Presentar la biografía de personajes peruanos como modelo de vida para incentivar en los estudiantes la reflexión de las propias metas y objetivos

\section{Competencias:}

- Los estudiantes identifican los detalles importantes y las ideas principales en un texto biográfico de uno de los máximos representantes de la literatura peruana.

- Analizan de forma crítica y reflexiva el accionar de un personaje, llevando a hacer comparaciones con la propia vida.

- Trabajan en parejas y en equipo respetando las ideas de los demás, sabiendo cuando intervenir y empleando lenguaje respetuoso y correcto.

- Hablar de los propios objetivos y sueños.

\section{1. ¿Quién es?}


Antes de empezar con la actividad se recomienda hacer una lluvia de ideas preguntando si conocen a César Vallejo. Escriba las respuestas de los estudiantes en la pizarra.

Empezar con la actividad 1. Dar unos 3 minutos de tiempo para desarrollar la actividad de forma individual, después formar parejas para verificar las respuestas. Después, pida a un estudiante que lea el texto completo y pregunte a la clase si están de acuerdo con la respuesta.

Respuesta: ver el texto al final de la guía.

\section{2. ¿Cómo era Vallejo?}

El objetivo de esta actividad es invitar a los estudiantes a ser observadores y formular hipótesis mientras se trabaja en pareja. Dar al menos 5 minutos para que la pareja llegue a una conclusión, después invite a uno de los integrantes a decir sus respuestas a la clase. Repita la acción con otros integrantes. Deje que sean los estudiantes usen la imaginación, no hay respuesta incorrecta.

\section{La biografía}

Es una actividad muy simple con el objetivo de hacer una lectura veloz. Dejar que los estudiantes trabajen de forma individual y luego en parejas. Después del trabajo en parejas invite a algunos estudiantes a leer en voz alta el texto y preguntar a la clase si las respuestas coinciden. Luego, confirme o niegue Usted la respuesta.

Respuesta: ver el texto al final de la guía.

\section{Leamos: verdadero o falso}

Dar unos dos minutos para que los estudiantes respondan, después invite a una verificación en parejas, y finalmente trabaje con toda la clase.

Respuesta: V-V-F-V-V

\section{5. ¡Sorprende!}

El objetivo es que se trabaje de forma crítica y reflexiva en función de la propia experiencia de vida, por ello cada estudiante es libre en su respuesta, el único requisito es saber sustentar su elección.

Después de un trabajo individual entre 5 y 8 minutos, forme grupos de 4 integrantes e invite a una discusión. Cada estudiante es libre de manifestar sus ideas. Recuerde que aquí se está trabajando el respeto por la opinión de los demás, el saber tomar la palabra y usar un lenguaje correcto. Para finalizar, si lo cree conveniente, se puede invitar a un delegado de cada grupo para exponer las conclusiones de cada grupo.

\section{Ventajas y desventajas}

Forme parejas y después pida que realicen la actividad sugerida. Uno de los objetivos de esta actividad es que los estudiantes puedan trabajar en parejas respetando las ideas y que lleguen a una conclusión. No hay respuesta equivocada. Para la corrección de la actividad, divida la pizarra en dos como el esquema de la actividad: positivo y negativo; luego invite a los alumnos a escribir sus respuestas.

\section{7. ¡Entrevista a Vallejo!}

Esta actividad permite ponerse en los zapatos del poeta y a hacer una lectura más profunda sobre su biografía. Forme parejas y conceda al menos 15 minutos para preparar la entrevista. Sugiera que deben usar la imaginación para las preguntas y las respuestas. Para una puesta en escena, puede invitar a los estudiantes a observar las fotos de la actividad 2, para imitar la postura del poeta peruano.

\section{Carta a Vallejo}

Esta actividad puede realizarse en el aula o en casa. Permite al estudiante a aflorar sus ideas respecto a sus metas y objetivos.

Distribuya hojas a los estudiantes (tienen que ser del mismo formato) y pídales que escriban la carta. Todos los estudiantes deben utilizar el mismo color de lapicero o lápiz. Indique, también, que no deben firmar la carta, debe ser anónima. Cuando hayan terminado de escribir (aprox. 20 min.) recoja las cartas y entrevérelas y distribúyelas. Cuando un estudiante haya terminado de leer una carta, debe intercambiarla con otro compañero. Se debe leer mínimo 5 cartas.

El hecho de no poner los nombres en las cartas es para que en el momento de la lectura no haya burlas o nadie se sientajuzgado. 


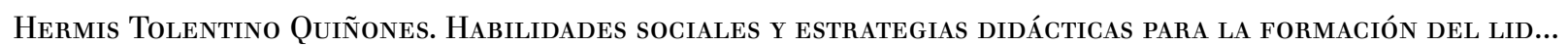

CC BY-NC-ND 